\title{
Análisis y Optimización del Proceso de Producción en una Empresa Procesadora de Leche
}

\author{
Josef Jablonsky y Veronika Skocdopolova \\ Facultad de Informática y Estadística, Dpto. de Econometría, Universidad de Economía, \\ Praga, República Checa (e-mail: jablon@vse.cz; skov01@vse.cz)
}

Recibido Dic. 5, 2016; Aceptado Feb. 8, 2017; Versión final Abr. 3, 2017, Publicado Ago. 2017

\begin{abstract}
Resumen
Este artículo describe un modelo para analizar el proceso de producción de una gran empresa internacional de procesamiento de leche, con el principal objetivo de minimizar los costos totales de producción. La optimización del proceso es una tarea importante que debe resolverse en la planificación estratégica y operativa de cada empresa industrial. La metodología propuesta visualiza la situación como un problema de optimización de mezcla lineal y el modelo se basa en la metodología de programación por metas. El modelo se implementó en una aplicación de software original que se utiliza actualmente como una herramienta en el proceso de toma de decisiones operativas en la empresa. Este sistema es una aplicación complementaria de MS Excel escrita en VBA (Visual Basic para Aplicaciones). La experiencia actual de los usuarios con la aplicación y sus resultados son positivos y la compañía está interesada en seguir desarrollándola.
\end{abstract}

Palabras clave: problema de mezcla; programación por metas; industria de procesamiento de leche; LINGO; MS Excel

\section{Analysis and Optimization of the Production Process in a Milk Processing Company}

\begin{abstract}
The paper presents a model to analyse the production process of a large international milk-processing company, with the main objective of minimizing total production costs. Process optimization is an important task that must be solved during the strategic and operating steps of the company planning. The proposed methodology visualizes the situations as a linear blending optimisation problem and the model is based on goal programming methodology. The model was implemented using an original software application that is currently used in the company as an operational decision making tool. This system is a MS Excel add-in application written in VBA (Visual Basic for Applications). The experience of the users that are currently using the software is positive and the company shows interest to continue using the application.
\end{abstract}




\section{INTRODUCCIÓN}

La optimización del proceso de producción es una tarea importante que debe resolverse en la planificación estratégica y/u operativa de cada empresa industrial. Hace algún tiempo, una gran empresa internacional de procesamiento de leche se dirigió a nuestro departamento con una petición para analizar su proceso de producción en las condiciones dadas con el objetivo de minimizar los costos totales. Este artículo describe el modelo formulado de acuerdo a las peticiones de esta compañía y a las experiencias de su uso en la planificación operativa. Por otro lado, el análisis tiene como objetivo, la optimización del llamado proceso de producción de "masa blanca". El término de "masa blanca" se utiliza como base para la producción de yogures, bebidas lácteas acidificadas y otros derivados de la leche. El problema que la empresa resuelve no solamente consiste en la mezcla de materiales básicos como la leche cruda y otros ingredientes lácteos sino también la optimización de la gestión de compras.

La planificación diaria de la producción es uno de los problemas más difíciles que enfrentan los directivos de la industria lechera. Estos han sido discutidos por muchos investigadores en el pasado. Sullivan y Secrest (1985) han analizado la implementación de un sistema de apoyo para la toma de decisiones basado en la optimización para planificar la producción en una empresa procesadora de leche. Doganis y Sarimveis (2007) han formulado modelos mixtos de programación lineal para la optimización de líneas de producción de yogur. En Andrade et al. (2010) se han estudiado varios factores que influyen en la calidad del producto final (yogur). Droste y Deuse (2012) han abordado un nuevo enfoque para planificar y optimizar los procesos de producción de leche en la planta. Este permite a los planificadores una reconfiguración más rápida de los sistemas de provisión de materiales. Granados et al. (2014) han discutido varios aspectos del procesamiento de la leche con respecto a la producción de queso.

Desde el punto de vista metodológico, este artículo se dedica a resolver un problema lineal de optimización de mezcla en el que una de las tareas más importantes es lograr una composición definida de masas blancas dentro de una tolerancia dada. El principal objetivo del problema es la minimización de los costos totales. En general, los problemas de mezcla pertenecen a uno de los más utilizados en los casos de optimización de problemas reales de producción. Es posible encontrar numerosas aplicaciones descritas en muchos artículos. Nuestro problema puede ser formulado por medio de la metodología de programación por metas. Similar enfoque se utilizó en muchos estudios en el pasado - Rehman y Romero (1987) y Romero y Rehman (2003) utilizan la programación por metas con funciones de penalización para las decisiones agrícolas. Jablonsky (1992) soluciona un problema de la nutrición diaria de pacientes en hospitales, Ferguson Et al. (2006) proponen el diseño de recomendaciones de alimentación complementaria para niños pequeños usando modelos de programación por metas. Bilgen y Oskarahan (2007) ofrecen un modelo de optimización para la mezcla de grano y su envío. Skocdopolova y Jablonsky (2009) publicaron un artículo que ofrece una solución de problemas de mezcla usando la programación por metas. Zgajnar et al. (2009) formulan un modelo de programación por metas para las raciones de vacas lecheras. Una extensa información sobre la teoría y la práctica de la programación por metas se puede encontrar en Jones y Tamiz (2010).

El objetivo principal de la producción de masa blanca es realizar la mezcla de acuerdo a diversas recetas y conseguir los costos mínimos de compra con respecto a las restricciones dadas. La empresa trabaja con varios tipos de materiales (por ejemplo, leche cruda, crema, leche desnatada, leche concentrada, leche en polvo y grasas lácteas anhidras). Estos se pueden adquirir para ser procesados en productos semifinales, usados para mezclar los productos finales, o directamente vendidos a los consumidores. Tenemos que tener en cuenta que la leche cruda no puede ser utilizada directamente como material para la mezcla de masa blanca. Se puede utilizar como un semi-producto para la leche desnatada y la producción de crema solamente. La propia compañía puede producir otras materias primas como la leche concentrada y la leche en polvo de la leche desnatada o de la grasa láctea anhidra de la crema. El esquema de producción y adquisición se presenta en la Figura 1.

El trabajo está organizado de la siguiente manera: la sección número dos contiene cuestiones metodológicas y la formulación del modelo. Debido a su complejidad, no todos los parámetros del modelo, variables y restricciones se describen en detalle. Todos los parámetros requeridos por la empresa están incluidos en el modelo. La siguiente sección contiene información acerca del software de aplicación OPTIPROT que la empresa utiliza en su planificación operativa. Esta aplicación ha sido escrita como complemento para MS Excel en cooperación con LINGO, que proporciona un entorno de modelado para construir, resolver y analizar modelos, junto a sus solucionadores lineales y enteros. La sección final del artículo discute las experiencias con el modelo y sus diferentes posibles extensiones. 


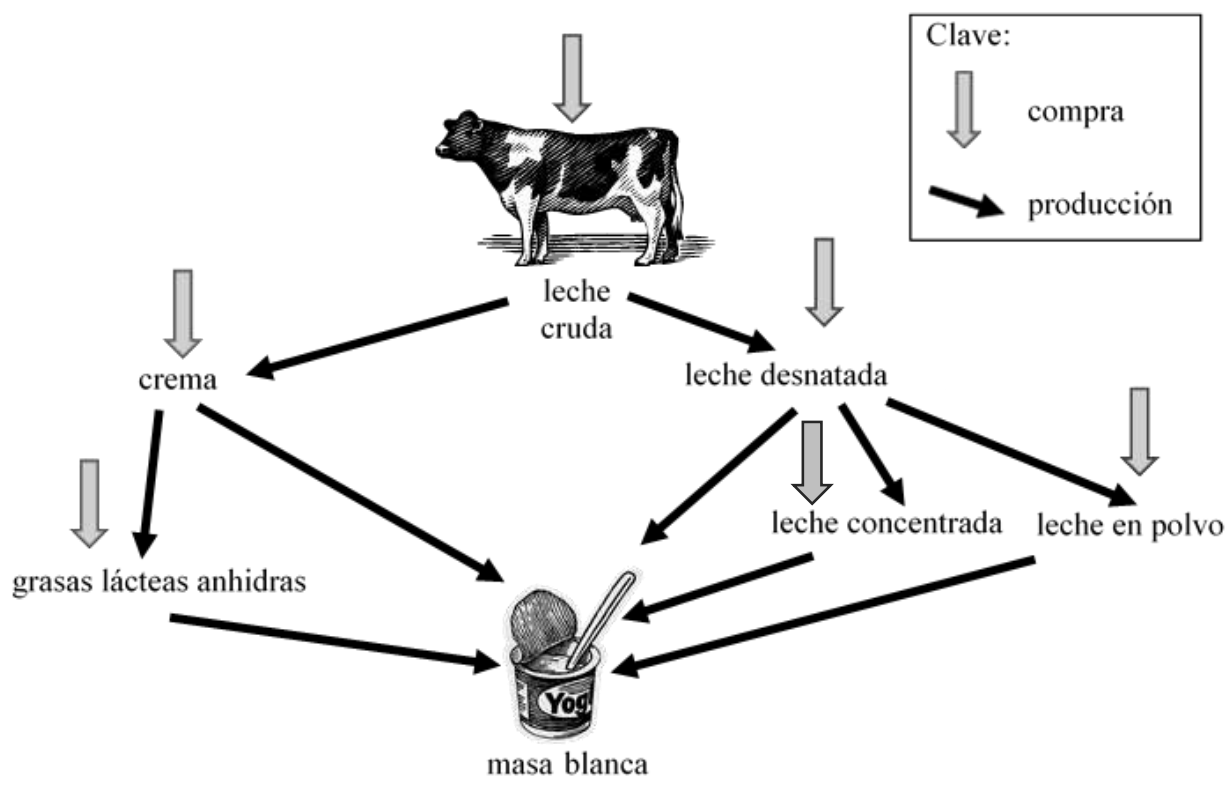

Fig.1: Esquema de la producción de masa blanca

\section{FORMULACIÓN DEL MODELO}

El modelo de optimización del problema se basa en la metodología de programación por metas con la excepción de que se utilizan algunas extensiones necesarias para respetar las solicitudes de la empresa. La programación por metas trabaja con metas flexibles e inflexibles. Las metas inflexibles son restricciones convencionales conocidas por la formulación general del problema de programación matemática. Estos deberían cumplirse sin cualquier tolerancia. Por el contrario, las metas flexibles no tienen que ser cumplidas exactamente, pero ciertas desviaciones positivas o negativas están permitidas. La función objetivo del modelo de programación por metas minimiza la suma de las desviaciones relativas o la desviación relativa máxima de los objetivos predefinidos. El modelo que minimiza la máxima desviación relativa se formula a continuación. Su modificación se utiliza en el modelo para la optimización del proceso de producción.

Minimizar

$\delta$

Sujeto a:

$\sum_{j=1}^{n} c_{k j} x_{j}+d_{k}^{-}-d_{k}^{+}=g_{k}, \quad k=1,2, \ldots, p$,

$w_{k}^{-} d_{k}^{-}+w_{k}^{+} d_{k}^{+} \leq \delta g_{i}, \quad k=1,2, \ldots, p$,

$\sum_{j=1}^{n} a_{i j} x_{j} \leq b_{i}$

$i=1,2, \ldots, m$

$d_{k}^{-}, d_{k}^{+} \geq 0$,

$k=1,2, \ldots, p$

donde

$n$ es el número de variables del modelo,

$p$ es el número de objetivos flexibles;

$m$ es el número de objetivos inflexibles;

$c_{k j}, k=1,2, \ldots, p, j=1,2, \ldots, n$ es el coeficiente que expresa la relación entre los objetivos flexibles $k$-ésimos y la variable $j$-ésima;

$g_{k}, k=1,2, \ldots, p$ es el valor del objetivo flexible $k$-ésimo;

$d_{k}{ }^{-}, d_{k^{+}}, k=1,2, \ldots, p$ es la negativa (positiva) variable de desviación que indica bajo- (sobre-) el logro del valor del objetivo $g_{k}$; 
$w_{k}{ }^{-}, w_{k}{ }^{+}, k=1,2, \ldots, p$, es el peso que expresa la importancia del logro del objetivo $k$-ésimo del objetivo asignado a variables de desviación (positivas) negativas;

$x_{j}, j=1,2, \ldots, n$ es la variable que expresa la cantidad del $j$-ésimo ingrediente que tenemos que mezclar, $\delta$ es la desviación relativa ponderada máxima.

La función objetivo (1) minimiza la desviación máxima relativa, las restricciones de balance (2) expresan las metas flexibles, las restricciones (3) expresan que todas las desviaciones relativas ponderadas son menores 0 iguales que $\delta$, (4) son metas inflexibles (5) las restricciones no-negativas. En nuestro contexto las metas inflexibles corresponden a las masas blancas y los requisitos de composición (grasa, proteínas, etc.), las variables $x_{j}$ expresan la cantidad del ingrediente j-ésimo (leche, crema, etc.) utilizados para la mezcla del producto determinado y la desviación de las variables $d_{k}{ }^{-}, d_{k}{ }^{+}$en $\%$ expresan las desviaciones de las recetas de las masas blancas. Por supuesto, el modelo completo propuesto es mucho más complejo y el modelo no minimiza la desviación máxima como aquí en (1), pero el principal criterio de optimización es la minimización de los costos totales.

Una formulación detallada del modelo matemático no es posible debido al elevado número de parámetros, limitaciones de diferente naturaleza y variables. A continuación se muestra al menos una breve descripción de las principales partes del modelo matemático sin utilizar ninguna notación formal. Aunque la formulación matemática no sea exacta y no ofrezca la suficiente información acerca de la estructura del modelo y sus principales componentes.

\section{Las restricciones}

El proceso de producción de la empresa y sus características particulares generan muchas restricciones que deben ser tomadas en cuenta en el modelo. Se pueden dividir en tres categorías principales. La primera categoría de restricciones se refiere a las materias primas más utilizadas para la producción de los productos semi-acabados o los productos finales. Cada posición de material se caracteriza, entre otros, por su contenido de grasa y proteína. Estas dos características son las más importantes. Deben tenerse en cuenta con el fin de asegurar que el contenido de grasa y proteína en los productos finales y/o semi-productos es la deseada. El balance de grasa y proteína es esencial para la crema y la producción de leche desnatada. La grasa y el equilibrio de proteínas también es importante para la producción de leche concentrada, el balance proteico es monitoreado en la producción de leche en polvo y la cantidad de grasa se controla durante la producción de grasa láctea anhidra de crema.

Las restricciones adicionales se derivan de la utilización de la leche en polvo. Es posible utilizar la leche en polvo como polvo o diluir la leche en polvo y luego usarla como leche desnatada, la cual se llama leche en polvo reconstituida. Por un lado es necesario también tener en cuenta la limitada cantidad dada de ingredientes para la producción de otras materias primas y por otro lado, la producción de masa blanca. Las cantidades de ingredientes producidas o compradas deben ser igual a la cantidad utilizada en la producción o la que se vende. Se puede expresar a grandes rasgos con el siguiente simple balance:

Adquisición (o producción) = uso de la producción de masa blanca + uso de la producción de otros materiales + venta de excedentes.

Los contratos de la compañía con algunos de sus vendedores requieren que cierta cantidad de leche y otro material que deben ser adquiridos. Estos montos mínimos o máximos de materiales generan muchas limitaciones adicionales al modelo.

La segunda categoría de limitaciones se refiere a las masas blancas que deben ser producidas de acuerdo a las recetas indicadas dentro de pequeñas tolerancias definidas. Las recetas de masas blancas se caracterizan por la grasa, el contenido de proteína y el contenido total de ingredientes lácteos en masas blancas. Las masas blancas nunca están compuestas por ingredientes $100 \%$ leche, siempre se complementan con otros componentes como los cultivos vivos de yogur, azúcares, o ingredientes de frutas. Excepto esto, las recetas de las masas blancas definen los límites en la proporción de grasa y proteína, lo que significa que la leche en polvo no puede ser un componente de algunas masas en absoluto, o que la única grasa en la masa blanca puede ser la grasa de la crema, etc. Otro ejemplo se refiere a una masa, en la que hasta el $50 \%$ de la grasa láctea anhidra puede ser utilizada. Esta importante restricción del modelo se deduce de las cantidades totales requeridas de masas blancas en toneladas que deben cumplirse exactamente. 
Las restricciones de la tercera categoría están relacionadas con las ventas de excedentes. De todos modos la empresa tiene la oportunidad de vender los excedentes de materias primas, incluyendo el exceso de leche cruda, pero sólo en importes máximos predeterminados. La cantidad sobrante se define como la diferencia entre la cantidad de materia prima adquirida (o producida) y la cantidad utilizada ya sea para producir otra materia prima o masa blanca. Al igual que con los vendedores de la compañía tiene contratos con sus clientes para suministrar cierta cantidad de materia prima a un precio determinado.

\section{Los parámetros}

Todos los datos necesarios para el modelo de optimización se introducen como sus parámetros de entrada. Estos parámetros describen con detalle las materias primas adquiridas (o producidas), las ventas de excedentes y las recetas de masas blancas. Cada uno de los materiales comprados se caracteriza por seis cifras - nombre del ingrediente, por ejemplo, la leche cruda, la crema, la leche desnatada, etc. (hay hasta ocho proveedores diferentes de cada ingrediente y cada uno de ellos puede entregar los ingredientes con una composición diferente en cuanto al contenido de grasa y proteína), el contenido de grasa y proteína de los ingredientes (en \%), su precio (en moneda local por kg), la cantidad mínima que debe ser adquirida por cada proveedor, y la cantidad máxima que se puede comprar. Todas estas seis cifras representan una oferta de cada proveedor; por ejemplo un proveedor ofrece crema con cierta composición a un precio determinado en una cierta cantidad máxima. Los materiales que son producidos por la empresa como los semi-productos (crema, leche desnatada, leche concentrada, leche en polvo y grasa láctea anhidra) se describen solamente por el contenido de grasa y proteína o por la mínima cantidad producida necesaria. La cantidad máxima producida depende de la cantidad disponible de los materiales necesarios para su producción. Los costos de semi-producción también son parámetros importantes del modelo. Esto significa los costos de procesamiento de $1 \mathrm{~kg}$ de leche cruda, los costos de producción de $1 \mathrm{~kg}$ de leche concentrada o en polvo y grasa láctea anhidra.

Del mismo modo los ingredientes adquiridos se refieren a las ofertas de los proveedores, las ventas de excedentes se relacionan con los requisitos de los clientes. Los clientes están dispuestos a pagar una cierta cantidad por $1 \mathrm{~kg}$ de ingredientes pero son capaces de comprar sólo una cantidad limitada. También pueden estar bajo contrato con la empresa para comprar una cierta cantidad mínima de cualquier ingrediente. Este contrato es mutuamente vinculante, lo que significa que el cliente tiene que comprar cierta cantidad de ingredientes y la empresa tiene que garantizar un suministro adecuado para satisfacer este requisito. La información sobre las características finales de los productos, como el contenido de grasa, proteínas e ingredientes lácteos es otro grupo importante de los parámetros del modelo. Las recetas de los productos finales son confidenciales y la compañía no permite su publicación. Es por ello que no podemos comentar este grupo de parámetros con más detalle.

Tabla 1: Los parámetros de entrada del modelo - ilustración

Parámetros de entrada periodo: Oct 2016

\begin{tabular}{|l|l|r|r|r|c|c|}
\hline & & $\begin{array}{c}\text { Proteína } \\
\text { (\%) }\end{array}$ & $\begin{array}{c}\text { Grasa } \\
\text { (\%) }\end{array}$ & $\begin{array}{c}\text { Precio } \\
(\text { ML/kg) }\end{array}$ & $\begin{array}{c}\text { Compromiso de } \\
\text { comprar/de producir (T) }\end{array}$ & $\begin{array}{c}\text { Posibilidad de } \\
\text { comprar/de producir (T) }\end{array}$ \\
\hline RM1 & Recoger & 3,53 & 3,95 & 8,10 & 5681 & 200 \\
\hline RM2 & Leche cruda 2 & 3,53 & 3,95 & 8,29 & & \\
\hline RM3 & Leche cruda 3 & 3,53 & 3,95 & 8,29 & & \\
\hline RM4 & Leche cruda 4 & 3,53 & 3,95 & 8,29 & & \\
\hline RM5 & Leche cruda 5 & 3,53 & 3,95 & 8,29 & & 500 \\
\hline RM6 & Leche cruda 6 & 3,53 & 3,95 & 8,29 & & 50 \\
\hline RM7 & Leche cruda 7 & 3,53 & 3,95 & 8,79 & & 50 \\
\hline CR1 & Crema producida & 1,95 & 40,00 & & & \\
\hline CR2 & Crema 2 & 1,95 & 40,00 & 28,78 & & \\
\hline CR3 & Crema 3 & 1,95 & 40,00 & 28,78 & & \\
\hline CR4 & Crema 4 & 1,95 & 40,00 & 28,78 & & \\
\hline CR5 & Crema 5 & 1,95 & 40,00 & 28,78 & & \\
\hline
\end{tabular}


Tabla 1 (continuación)

Venta del excedente

\begin{tabular}{|l|l|c|c|c|}
\hline & & Precio (ML/kg) & $\begin{array}{c}\text { Compromiso de } \\
\text { vender (T) }\end{array}$ & $\begin{array}{c}\text { Posibilidad de } \\
\text { vender (T) }\end{array}$ \\
\hline $\mathrm{RM}(1)$ & Leche cruda (1) & 7,10 & & 25 \\
\hline $\mathrm{RM}(2)$ & Leche cruda (2) & 6,90 & & 100 \\
\hline $\mathrm{RM}(3)$ & Leche cruda (3) & 6,70 & & \\
\hline $\mathrm{RM}(4)$ & Leche cruda (4) & 6,50 & & 1000 \\
\hline $\mathrm{RM}(5)$ & Leche cruda (5) & 6,30 & & 25 \\
\hline $\mathrm{RM}(6)$ & Leche cruda (6) & 6,10 & & 25 \\
\hline $\mathrm{CR}(1)$ & Crema (1) & 27,34 & & 50 \\
\hline $\mathrm{CR}(2)$ & Crema (2) & 26,84 & & \\
\hline $\mathrm{CR}(3)$ & Crema (3) & 26,34 & & \\
\hline $\mathrm{CR}(4)$ & Crema (4) & 25,84 & & \\
\hline $\mathrm{CR}(5)$ & Crema (5) & 25,34 & & \\
\hline
\end{tabular}

Costo de producción

\begin{tabular}{|c|c|c|c|}
\hline $\begin{array}{l}\text { Tratamiento de la leche (pasteurización, } \\
\text { desnatado) }\end{array}$ & & $\mathrm{ML} / \mathrm{kg}$ & 0,40 \\
\hline Producción de SMC (evaporación) & & $\mathrm{ML} / \mathrm{kg}$ & 2,00 \\
\hline Producción SMP & & $\mathrm{ML} / \mathrm{kg}$ & 12,00 \\
\hline AMF/producción de mantequilla & & $\mathrm{ML} / \mathrm{kg}$ & 8,00 \\
\hline Desviación positiva & $4,0 \%$ & & \\
\hline
\end{tabular}

La Tabla 1 muestra la interfaz de MS Excel para introducir los parámetros de entrada de las dos primeras categorías (ingredientes comprados y la venta de excedentes). La segunda columna de cada una de las tablas contiene los proveedores o los nombres de los clientes. El precio se expresa en Moneda Local (ML). Los parámetros de entrada para las recetas de masas blancas están en otra hoja de la aplicación y no se pueden presentar debido a su confidencialidad.

\section{Las variables}

Hay cientos de variables en la versión actual del modelo. La mayor parte de ellas expresan la cantidad de cada ingrediente utilizado en la producción de cada masa blanca (hasta 30 masas blancas y cerca de 40 ingredientes). Otras variables representan la cantidad de cada ingrediente que debe ser adquirida de cada proveedor y revendida a cada cliente. En el modelo también hay variables definidas que muestran cantidad de ingredientes y variables que denotan la cantidad de material utilizado para la producción de otros materiales producidos.

\section{La función objetivo}

La función objetivo del modelo expresa la minimización de los costos totales del proceso de producción. La función objetivo se compone de las siguientes principales partes: i) Los costos de la leche cruda y otros ingredientes adquiridos; ii) Los costos de producción de crema, leche desnatada, leche concentrada, leche en polvo y grasa láctea anhidra; iii) Los ingresos por ventas de excedentes; iv) El valor de las inventarios inicial; y v) El valor final de los inventarios. El modelo completo contiene más de 1500 variables (algunos de ellas son números enteros) y casi 500 restricciones. No es posible resolver el modelo utilizando el complemento de Excel Solver debido a sus limitaciones en el número de restricciones y variables. Por tal motivo se usa un Solver lineal externo y entero. El sistema de modelado LINGO se incorpora a la aplicación dado su costo aceptable y suficiente potencia. Los detalles sobre este sistema y sobre su lenguaje de modelado se pueden encontrar en Schrage (2007). La Tabla 2 resume los principales resultados.

Debido al hecho de que la compañía no solamente opera en la República Checa, la aplicación puede ser utilizada en diferentes países de Europa (incluso no europeos). Las fábricas en cada país tienen diferentes instalaciones técnicas y usan diferentes procedimientos. Este hecho se tiene en cuenta para el ajuste de los parámetros de la aplicación. Esta parte contiene las desviaciones de configuración para la composición de los productos finales, la posibilidad de crear valores, o la necesidad de usar ingredientes de los inventarios, con la excepción de las posibilidades de restricción de la producción de algunos de los ingredientes. 
Tabla 2: Informe de los resultados - ilustración

Compra

periodo:

\begin{tabular}{|c|c|c|c|c|c|c|c|c|c|}
\hline & & \multirow{3}{*}{$\begin{array}{c}\text { Compromiso } \\
\text { de comprar / de } \\
\text { producir }(T)\end{array}$} & \multirow{3}{*}{$\begin{array}{c}\text { Recomendación } \\
\text { para comprar / } \\
\text { para producir }(T)\end{array}$} & \multicolumn{6}{|c|}{ Consumo } \\
\hline & & & & \multirow{2}{*}{$W M$} & \multirow{2}{*}{ Revender } & \multicolumn{2}{|c|}{ CR para SM para } & \multirow{2}{*}{$\begin{array}{c}\text { SM para } \\
\text { SMP }\end{array}$} & \multirow{2}{*}{$\begin{array}{c}\text { SMP para } \\
\text { RECON }\end{array}$} \\
\hline & & & & & & $A M F$ & $S M C$ & & \\
\hline RM1 & Recoger & 5681 & 0 & & 125 & & & & \\
\hline RM2 & Leche cruda 2 & 0 & 0 & & 0 & & & & \\
\hline RM3 & Leche cruda 3 & 0 & 0 & & 0 & & & & \\
\hline RM4 & Leche cruda 4 & 0 & 0 & & 0 & & & & \\
\hline RM5 & Leche cruda 5 & 0 & 0 & & 0 & & & & \\
\hline RM6 & Leche cruda 6 & 0 & 0 & & 0 & & & & \\
\hline RM7 & Leche cruda 7 & 0 & 0 & & 0 & & & & \\
\hline CR1 & Crema producida & 0 & 542 & 489 & 54 & 0 & & & \\
\hline CR2 & Crema 2 & 0 & 0 & 0 & 0 & 0 & & & \\
\hline CR3 & Crema 3 & 0 & 0 & 0 & 0 & 0 & & & \\
\hline
\end{tabular}

Revender

\begin{tabular}{|l|l|c|c|}
\hline & & $\begin{array}{c}\text { Compromiso } \\
\text { de vender }(T)\end{array}$ & $\begin{array}{c}\text { Recomendación } \\
\text { para vender }(T)\end{array}$ \\
\hline $\mathrm{RM}(1)$ & Leche cruda (1) & 0 & 25 \\
\hline $\mathrm{RM}(2)$ & Leche cruda (2) & 0 & 0 \\
\hline $\mathrm{RM}(3)$ & Leche cruda (3) & 0 & 100 \\
\hline $\mathrm{RM}(4)$ & Leche cruda (4) & 0 & 0 \\
\hline $\mathrm{RM}(5)$ & Leche cruda (5) & 0 & 0 \\
\hline $\mathrm{RM}(6)$ & Leche cruda (6) & 0 & 0 \\
\hline $\mathrm{CR}(1)$ & Crema (1) & 0 & 25 \\
\hline $\mathrm{CR}(2)$ & Crema (2) & 0 & 25 \\
\hline $\mathrm{CR}(3)$ & Crema (3) & 0 & 4 \\
\hline $\mathrm{CR}(4)$ & Crema (4) & 0 & 0 \\
\hline $\mathrm{CR}(5)$ & Crema (5) & 0 & 0 \\
\hline $\mathrm{CR}(6)$ & Crema (6) & 0 & 0 \\
\hline $\mathrm{SM}(1)$ & Leche desnatada (1) & 0 & 0 \\
\hline $\mathrm{SM}(2)$ & Leche desnatada (2) & 0 & 0 \\
\hline
\end{tabular}

\begin{tabular}{|c|c|}
\hline Costo total: & \\
\hline 55743543 & $\mathrm{ML}$ \\
\hline \multicolumn{2}{|l|}{ Compra de leche cruda: } \\
\hline 46042071 & $\mathrm{ML}$ \\
\hline \multicolumn{2}{|l|}{ Compra de otros productos: } \\
\hline 5042150 & $\mathrm{ML}$ \\
\hline \multicolumn{2}{|l|}{ Costo de producción: } \\
\hline 2359201 & $\mathrm{ML}$ \\
\hline \multicolumn{2}{|l|}{ Venta del excedente: } \\
\hline 2300121 & $\mathrm{ML}$ \\
\hline \multicolumn{2}{|l|}{ Existencias iniciales: } \\
\hline 0 & $\mathrm{ML}$ \\
\hline \multicolumn{2}{|l|}{ Existencias de cierre: } \\
\hline 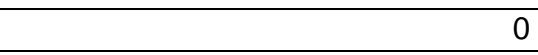 & $\mathrm{ML}$ \\
\hline
\end{tabular}

El informe con los resultados de la optimización brinda las recomendaciones a los usuarios para la adquisición de los ingredientes, la mezcla de los productos finales, y la producción o la reventa de excedentes. En el informe de los resultados (la hoja de MS Excel con este informe, se presenta en la Figura 3) se leen las cantidades (en toneladas) de cada ingrediente comprado (o producido) que se ha utilizado para la producción de otros ingredientes, para la producción de masas blancas, o la cantidad de ingredientes que se revende como excedente. En el caso de la leche en polvo, se encuentra la información acerca de la cantidad de leche utilizada en forma de polvo y, como leche reconstituida. Además, en el informe de resultados se encuentran los costos específicos de todo el proceso de producción. Los usuarios pueden ver los costos totales que se estructuran en secciones principales como se describió anteriormente.

Los resultados del modelo son bastante sensibles en la configuración de los parámetros. Si no se permiten las desviaciones en la composición de masas blancas o estas desviaciones son demasiado pequeñas, el modelo no tiene ninguna solución factible. La necesidad de utilizar las desviaciones distintas de cero se da principalmente de las desviaciones que miden el contenido de grasa y proteína en la leche cruda y otros ingredientes comprados. La aplicación contiene actualmente un mecanismo de prueba simple que señala la masa blanca, para la cual la desviación es insuficiente. Este mecanismo puede ayudar a los usuarios a identificar fuentes de inviabilidad y cambiar los parámetros del modelo con el fin de obtener una solución óptima fácilmente.

\section{CONCLUSIONES}

La aplicación OPTIPROT se utiliza actualmente en la planificación operativa de la filial checa de la compañía y se la ha probado en varias de sus sucursales en el extranjero. Se espera su posterior distribución a otros países europeos o incluso a países no europeos. Los resultados de sus pruebas son muy prometedores y la compañía está interesada en seguir desarrollando el sistema. Las principales direcciones de su desarrollo y mejoramiento pueden ser señaladas de la siguiente manera (algunas de las características mencionadas a continuación ya han sido aprobadas o están bajo desarrollo): 
(i) La primera versión de la aplicación planifica la producción dentro de un período solamente. Una de las principales peticiones de la empresa era la posibilidad de utilizar los resultados del proceso de optimización de un período como los parámetros de entrada del período siguiente. Este aspecto es importante cuando la empresa crea inventarios de algunos ingredientes y espera utilizarlos durante el próximo período. Esta opción se incluye en la versión actual de la aplicación y ya ha sido probada.

(ii) La comparación de los diversos escenarios propuestos por diferentes conjuntos de datos de entrada. Los diferentes conjuntos de parámetros de entrada conducen a diferentes óptimos resultados finales. Una herramienta que permite su comparación es necesaria para los administradores a fin de adoptar decisiones estratégicas.

(iii) La optimización de más plantas de producción al mismo tiempo. En este caso cabe preguntarse si dicho modelo sería un modelo lineal de criterio único y es más bien un punto de discusión y extensión del futuro sistema.

(iv) La cuantificación de ahorros y otras contribuciones presentadas por la aplicación OPTIPROT. Es muy difícil comparar el enfoque intuitivo pasado con la recomendación hecha por el sistema de optimización.

El modelo presentado para la planificación operativa en la gran empresa de procesamiento de leche demuestra que un modelo bastante sencillo de optimización lineal puede ayudar significativamente en la planificación de la producción, generar ahorros para la empresa y mejorar su capacidad para competir en el entorno económico. La experiencia actual de los usuarios con la aplicación y sus resultados son positivos y la compañía está interesada en seguir desarrollándola.

\section{AGRADECIMIENTOS}

Esta investigación se realizó con el apoyo de la agencia de subvenciones interna de la Facultad de Informática y Estadística, proyecto $N^{\circ}$ : F4/62/2015.

\section{REFERENCIAS}

Andrade, R. D., M.R. Arteaga y M. M. Simanca, Effect of the bran wheat on the rheological behavior of yogurt buffalo milk, Información Tecnológica, 21(5), 117-124 (2010)

Bilgen, B. y I. Ozkarahan, A mixed-integer linear programming model for bulk grain blending and shipping, International Journal of Production Economics, 107 (2), 555-571 (2007)

Doganis, P. y H. Sarimveis, Optimal scheduling in a yogurt production line based on mixed integer linear programming, Journal of Food Engineering, 80 (2), 445-453 (2007)

Droste M. y J. Deuse, A planning approach for in-plant milk run processes to optimize material provision in assembly systems. In: EIMaraghy $\mathrm{H}$. (Eds.). Enabling Manufacturing Competitiveness and Economic Sustainability, Springer, Berlin, Heidelberg (2012)

Ferguson, E.L., N. Darmon, y otros 4 autores, Design of optimal food-based complementary feeding recommendations and identification of key "problem nutrients" using goal programming, Journal of Nutrition, 136(10), 2399-2404 (2006)

Granados, C., L. A. Meza, R.S. Paba y D. Acevedo, Layer Cheese made from Buffalo Milk in The Municipality of Carmen de Bolívar (Colombia), Información Tecnológica, 25(6), 39-44 (2014)

Jablonsky J., Decision support system for prescribing of patient nutrition: An interactive AHP/goal programming approach. Proceedings of the 9th International Conference: Theory and Applications in Business, Industry and Government, Springer-Verlag, New York, 135-148 (1992)

Rehman T. y C. Romero, Goal programming with penalty functions and livestock ration formulation, Agricultural Systems, 23 (2), 117-132 (1987)

Romero, C. y T. Rehman, Multiple Criteria Analysis for Agricultural Decisions, Elsevier, Amsterdam (2003)

Schrage, L., Optimization Modeling with LINGO, Lindo Systems, Inc., Chicago (2007)

Skocdopolova, V. y J. Jablonsky, Optimisation of production process in food-processing company, In: Mathematical Methods in Economics 2009. Czech University of Life Sciences, Praga, 290-295 (2009)

Sullivan, R. S. y S. C. Secrest, A Simple Optimization DSS for Production Planning at Dairyman's Cooperative Creamery Association, Interfaces, 15(5), 46-53 (1985)

Jones, D., y M. Tamiz, Practical Goal Programming. International Series in Operations Research \& Management Science 141, Springer, New York (2010)

Zgajnar, J., L. Juvancic y S. Kavici, Combination of linear and weighted goal programming with penalty function in optimization of a daily dairy cow ration, Agricultural Economics, 55(10), 492-500 (2009) 